\title{
Circulação Coronária Dependente do Ventrículo Direito na Atresia Pulmonar com Septo Interventricular Íntegro. Ausência da Origem das Artérias Coronárias da Aorta
}

\author{
Ivan Romero Rivera, Valdir Ambrósio Moises, Celia Camelo Silva, Pedro Abujamra, \\ José Lázaro Andrade, Antonio Carlos Carvalho
}

São Paulo, SP

\begin{abstract}
São descritos os aspectos clínicos, ecocardiográficos e angiográficos de um neonato de sexo masculino, com cinco dias de vida e diagnóstico de atresia pulmonar com septo interventricular integro. Tanto o ecocardiograma como a aortografia mostraram ausência da origem das artérias coronárias da aorta. O ecocardiograma bidimensional e, posteriormente, a ventriculografia direita identificaram as artérias coronárias, originando-se no ventrículo direito. Não houve contrastação retrógrada da aorta ou do tronco pulmonar quando contrastadas as artérias coronárias. Este é o primeiro caso relatado com diagnóstico ecocardiográfico pré angiografia, e é um exemplo da necessidade de se avaliar as artérias coronárias em pacientes com atresia pulmonar e septo ventricular integro.
\end{abstract}

\author{
Right Ventricle-Dependent Coronary Circu- \\ lation in Pulmonary Atresia with Intact \\ Ventricular Septum. Absence of Origin of the \\ Coronary Arteries from the Aorta
}

This report describes the clinical, echocardiographic and angiographic aspects of a five-day old boy with pulmonary atresia and intact ventricular septum. Both the echocardiogram and the aortography did not show any coronary arteries arising from the aorta. Twodimensional echocardiography was able to identify the coronary arteries originating from the right ventricle and so did the right ventricular angiogram. No retrograde flow into the aorta or pulmonary trunk was identified after opacification of the coronary arteries. As far as we know this is the first case diagnosed by echocardiography, and is a vivid example of the necessity of identifying the coronary arteries in patients with pulmonary atresia and intact ventricular septum.
Comunicações entre as artérias coronárias e o ventrículo direito(VD) podem ser detectadas em, aproximadamente, $50 \%$ dos casos de atresia pulmonar com septo íntegro ${ }^{1-4}$. Porém, a ausência de conexão das artérias coronárias com a aorta, é extremamente rara, com poucos casos relatados na literatura ${ }^{6-8}$, todos diagnosticados mediante cateterismo ou autópsia. Apresentamos um caso desta rara anomalia, com diagnóstico ecocardiográfico e confirmação angiográfica.

Escola Paulista de Medicina - UNIFESP

Correspondência: Antonio Carlos Carvalho - Av. Jandira, 731/23 - 04080-004 São Paulo, SP

Recebido para publicação em 14/5/98

Aceito em 17/6/98

\section{Relato do caso}

Neonato de sexo masculino, pesando $3500 \mathrm{~g}$, nascido de parto normal, sem antecedentes pré-natais, foi transferido para nossa instituição no $5^{\circ}$ dia de vida com hipótese diagnóstica de atresia pulmonar com septo interventricular (SIV) íntegro. O exame físico revelou cianose importante, taquicardia e taquipnéia, pulsos presentes e simétricos e fígado palpável a $2 \mathrm{~cm}$ do rebordo costal direito; ausculta cardíaca de $2^{a}$ bulha única, normofonética, sem sopros, e ausculta pulmonar normal. A saturação arterial de oxigênio era de $50 \%$ em ar ambiente.

A radiografia de tórax mostrou área cardíaca normal e hipofluxo pulmonar bilateral e o eletrocardiograma(ECG) ritmo sinusal, eixo elétrico a $100^{\circ}$, sobrecarga atrial direita e ausência de potenciais elétricos do VD. 


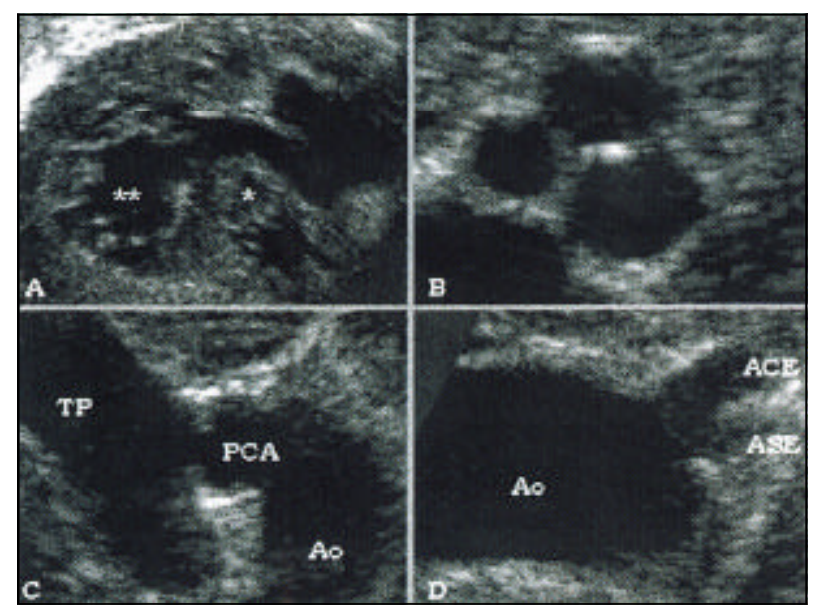

Fig. 1 - Dados ecocardiográficos mostrando: A) valvas mitral (**) e tricúspide $(*)$ na diástole; B) a valva pulmonar no corte transversal mostrando as três cúspides $\left({ }^{*}\right)$ aderidas entre si; C) a persistência do canal arterial (PCA), com estreitamento na extremidade pulmonar; D) a origem comum das artérias carótida esquerda (ACE) e subclávia esquerda (ASE). TP- tronco pulmonar; Ao- Aorta.

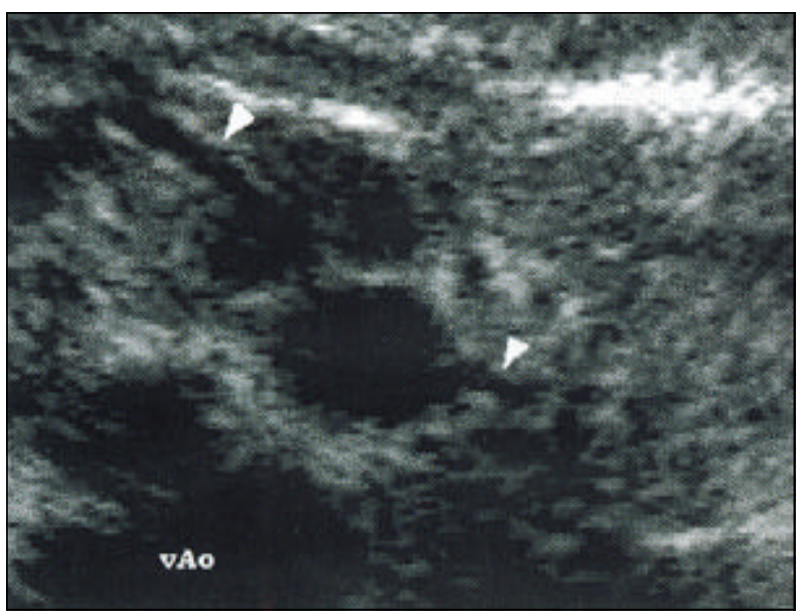

Fig. 2 - Imagem ecocardiográfica paraesternal transversal alta, mostrando a origem das duas artérias coronárias (pontas de flecha) na região imediatamente inferior à valva pulmonar. vAo- valva aórtica.

O ecocardiograma mostrou situs solitus, concordâncias atrioventriculare ventrículo-arterial, além de ampla comunicação interatrial tipo ostium secundum com fluxo laminar direita-esquerda ao estudo com Doppler e mapeamento de fluxo a cores. Uma imagem atravessando o interior do átrio direito em direção ao septo interatrial, sem obstrução ao fluxo, foi identificada como uma valva de Eustáquio exuberante. As valvas tricúspide e mitral foram melhor visibilizadas pelo corte subcostal e apresentavam áreas de $0,19 \mathrm{e} 1,22 \mathrm{~cm}^{2}$ e circunferências de $1,6 \mathrm{e} 4,2 \mathrm{~cm}$, respectivamente (fig. 1A).

As câmaras esquerdas apresentavam tamanho normal, com os parâmetros de função sistólica global e regional do ventrículo esquerdo (VE) preservados e com sinais de alteração do relaxamento diastólico ao estudo com Doppler pulsátil. O VD apresentava hipertrofia importante, sem contração sistólica evidente, sendo constituído quase exclusivamente pelas vias de entrada e de saída, esta última com hipoplasia moderada. A porção trabecular não foi bem identificada, existindo porém, múltiplos sinusóides no local.

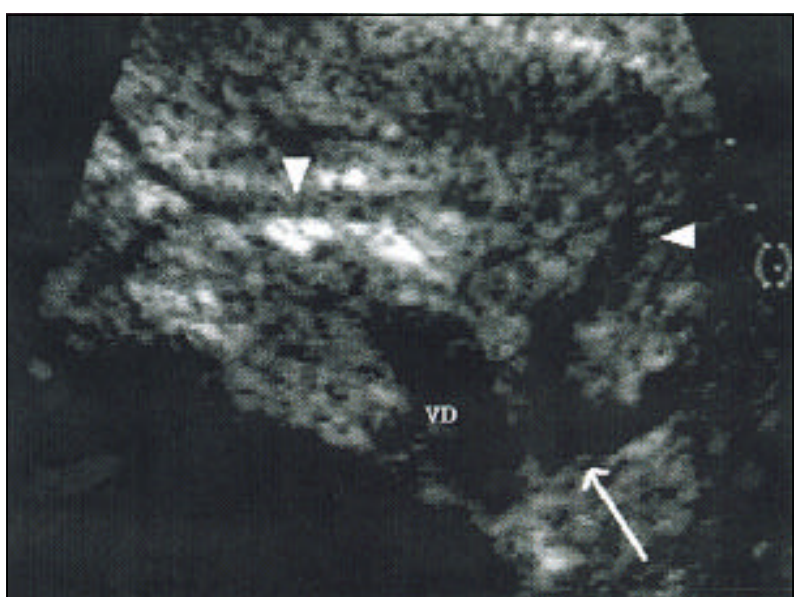

Fig. 3 - Imagem ecocardiográfica subcostal mostrando a artéria descendente anterior (pontas de flecha), desde a sua origem em espaço lacunar (flecha) até a sua bifurcação no ápex cardíaco. VD- ventrículo direito.

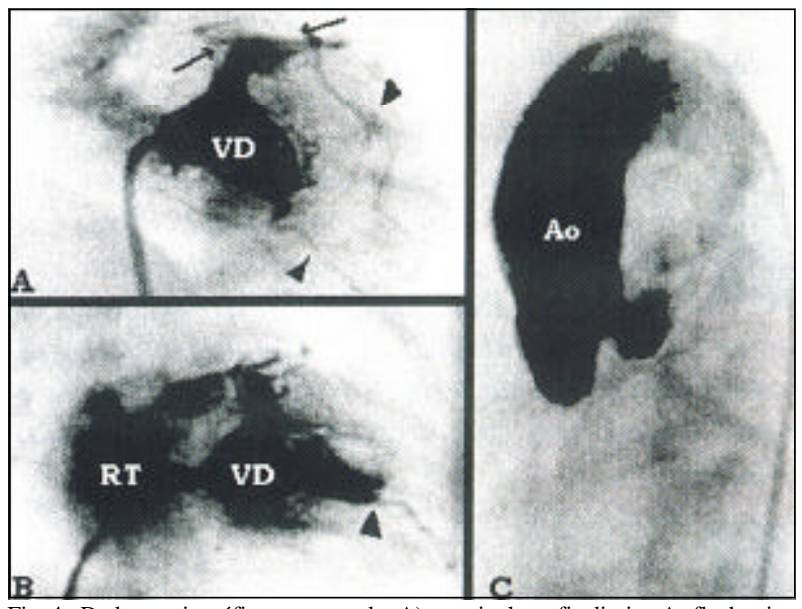

Fig. 4 - Dados angiográficos mostrando: A) ventriculografia direita. As flechas indicam a origem das artérias coronárias na via de saída do ventrículo direito (VD), imediatamente abaixo da valva pulmonar. A artéria descendente anterior é bem visibilizada (pontas de flecha), com o ponto de bifurcação identificado no ecocardiograma e a anastomose com um sinusóide na região apical do VD; B) ventriculografia direita, mostrando o refluxo tricúspide (RT). A ponta de seta demonstra o ponto de união da artéria coronária com um sinusóide; C) aortografia demonstrando ausência das artérias coronárias. Ao- aorta ascendente.

A valva pulmonar foi bem visibilizada no seu diâmetro transversal (fig. 1B), sendo constituída por três cúspides bem formadas e aderidas entre si; aderência também identificada na imagem longitudinal da tronco da artéria pulmonar como um tecido central de, aproximadamente, $5 \mathrm{~mm}$ de extensão no seu interior. Não havia fluxo anterógrado, sendo o fluxo arterial pulmonar totalmente dependente da persistência do canal arterial, que apresentava $4 \mathrm{~mm}$ de diâmetro na sua origem; na extremidade pulmonar, porém, apresentava uma redução de $50 \%$ do seu diâmetro (fig. 1C). O anel valvar e o tronco da artéria pulmonar mediam $8 \mathrm{~mm}$, os ramos pulmonares $5 \mathrm{~mm}$ cada um e a raiz aórtica $14 \mathrm{~mm}$ no diâmetro transversal.

O arco aórtico estava localizado à esquerda do tórax. Após a origem do tronco braquiocefálico, as artérias carótida e subclávia esquerdas apresentavam uma origem comum(fig. 1D). 
Apesar de exaustiva procura, nenhuma artéria coronária foi identificada nos seios coronários aórticos. Duas artérias coronárias foram vistas originando-se na via de saída do VD (fig. 2). Uma delas originava-se imediatamente abaixo da cúspide anterior direita da valva pulmonar; sendo esta coronária melhor visibilizada na imagem longitudinal do VD. Nesse corte ecocardiográfico, a via de saída do VD apresentava uma imagem lacunar, a partir da qual a artéria coronária se dirigia para a região ântero-inferior, apresentando bifurcação no terço distal da mesma (fig. 3). A outra coronária originava-se imediatamente abaixo da cúspide posterior da valva pulmonar, apresentando bifurcação após um curto trajeto. O estudo com Doppler pulsátil demonstrou fluxo sistólico nas artérias coronárias com gradiente máximo de $70 \mathrm{mmHg}$ na origem. O mapeamento de fluxo a cores não mostrou nenhuma anormalidade no local, mostrando porém, aceleração do fluxo na região apical do VD.

Com os dados ecocardiográficos, o paciente foi submetido a tratamento cirúrgico paliativo mediante cirurgia de Blalock-Taussig modificada à direita, com interposição de tubo de Goretex de 4mm. Houve boa evolução pós-operatória imediata e a gasimetria arterial mostrou saturação de oxigênio de $82 \%$ em ar ambiente, sendo dada alta hospitalar em bom estado geral.

Um mês após a alta hospitalar foi realizado estudo angiográfico (fig. 4) que confirmou a ausência das artérias coronárias a partir da aorta quando esta foi contrastada. A ventriculografia direita mostrou moderado a importante refluxo da valva tricúspide. A origem das artérias coronárias na via de saída do VD, visibilizada ao ecocardiograma, foi confirmada. Uma destas artérias, identificada como a artéria descendente anterior esquerda, apresentava múltiplas comunicações ventrículo-arteriais em todo o seu trajeto e, após contornar a região apical do VE, continuava-se com o mais apical dos sinusóides que constituíam a porção trabecular do VD, muitos dos quais terminando em fundo cego. A outra artéria, com origem na via de saída do VD, apresentava trifurcação, imediatamente após sua origem: um dos ramos contornando, posteriormente, a artéria pulmonar, anastomosando-se com a artéria descendente anterior; o outro ramo dirigindo-se para a região atrioventricular, formando uma discreta dilatação aneurismática, aparentemente, terminando em fundo cego e $\mathrm{o} 3^{\circ}$ ramo dirigindo-se em direção à parede anterior do VD. Não houve contrastação retrógrada da aorta ascendente nem do tronco pulmonar em nenhum momento, e o retorno venoso das coronárias foi bem identificado, drenando no seio venoso coronário.

Foi então indicado transplante cardíaco, estando o paciente no momento à espera de tratamento cirúrgico.

\section{Discussão}

Aproximadamente, 50\% dos casos de atresia pulmonar e SIV íntegro apresentam comunicação entre as artérias coronárias e o $\mathrm{VD}^{1-4}$, existindo uma correlação inversa das anomalias coronárias com a circunferência da valva tricús- pide e o tamanho das vias de entrada e de saída do $\mathrm{VD}^{2}$. Foi postulado que a comunicação se realiza mediante a persistência de conexões ventrículo-arteriais embriogênicas, que permitem o efluxo de sangue, a partir do VD hipertenso na sístole, criando um shunt circular, que passa do VD para as artérias coronárias, seio venoso coronário, átrio direito e, novamente, o VD ${ }^{1}$.

Varias anomalias das artérias coronárias foram identificadas na atresia pulmonar com SIV íntegro, entre as quais, presença de comunicações ventrículo-arteriais, sinusóides, fibrose, obstrução e interrupção das artérias coronárias ${ }^{2,4}$. A estenose ou interrupção das artérias coronárias pode estar localizada na região proximal ou distal à comunicação com o VD, existindo relato de alguns casos em que a porção proximal das artérias coronárias não pôde ser adequadamente visibilizada, tornando-se difícil, portanto, a diferenciação entre obstrução proximal e ausência do segmento proximal ${ }^{3}$. Nos casos de obstrução proximal, a circulação coronária é totalmente dependente do VD.

A ausência do segmento proximal das artérias coronárias, com sua origem na massa ventricular direita, é extremamente rara. Após o relato inicial de Lenox e Briner em $1972^{6}$, dois casos desta malformação foram adequadamente documentados em pacientes com atresia pulmonar e SIV íntegro ${ }^{7,8} \mathrm{e} \mathrm{um} \mathrm{caso} \mathrm{em} \mathrm{paciente} \mathrm{com} \mathrm{tetralogia} \mathrm{de} \mathrm{Fallot} \mathrm{e}$ hipoplasia importante do VD ${ }^{9}$. Em nenhum desses casos o diagnóstico foi realizado mediante ecocardiografia, o que torna nosso relato interessante e único na literatura.

O mecanismo embriológico desta malformação aindaé desconhecido ${ }^{5-8}$. A região proximal das artérias coronárias deriva diretamente da aorta, aproximadamente na $7^{\mathrm{a}}$ semana de gestação e se anastomosa, posteriormente, com os vasos originados da superfície epicárdica ${ }^{5,6}$, não sendo, portanto, um mecanismo diretamente relacionado com a persistência de comunicações ventrículo-arteriais ou sinusóides.

No nosso paciente, tanto o ecocardiograma como a angiografia aórtica demonstraram ausência da porção proximal das artérias coronárias, sem nenhuma evidência de contraste indo em direção à aorta, quando as artérias coronárias foram contrastadas, não se tratando, portanto, de interrupção das mesmas após a sua origem. Kasznica e $\mathrm{col}^{3}$ demonstraram ausência dos óstios coronários em um feto de 23 semanas de gestação, onde o segmento inicial das artérias coronárias estava presente, "semelhante a uma corda" de tecido, por fibrose extensa, com um mínimo lume. Apesar deste caso ser diferente do relatado por Lenox e Briner ${ }^{6}$, onde nenhum segmento inicial foi encontrado, não sabemos se, após o nascimento, o pequeno lume do vaso permitiria a passagem de contraste em direção retrógrada à aorta. A presença de múltiplas comunicações ventrículo-arteriais com a artéria descendente anterior é fato freqüentemente citado, assim como a presença de sinusóides intracardíacos terminando em fundo cego ${ }^{1}$. Embora as comunicações ventrículo-arteriais constituam verdadeiros vasos comunicantes, os sinusóides intracardíacos constituem espaços vasculares intramurais anômalos, produzindo um padrão trabeculado no miocárdio ${ }^{1,2}$. 
Um fato interessante, neste caso, é a ausência de manifestações isquêmicas em repouso, tanto no ECG como no ecocardiograma. Dos casos previamente relatados, dois apresentavam esta condição, sem evidência de alterações eletrocardiográficas ${ }^{7}$ ou anatômicas de necrose nos estudos de necropsia ${ }^{7,8}$.

O diagnóstico destas comunicações vasculares é extremamente importante na indicação cirúrgica de pacientes com atresia pulmonar, já que a descompressão do VD poderia determinar hipoperfusão coronária e acentuação da isquemia miocárdica. A realização de cirurgia de BlalockTaussig nesta criança visou, apenas, protegê-la das crises de hipóxia. Nestes casos onde a intervenção cirúrgica é realizada sem angiografia é fundamental que não se tente reali- zar abertura da valva pulmonar, pois se corre o risco, quando efetuada, de hipoperfusão coronária. Por outro lado é fundamental que o estudo angiográfico seja feito logo, pois, a documentação de uma circulação coronária VD-dependente está associada a maior risco de óbito ${ }^{1}$, inclusive com morte súbita, havendo nestes casos indicação indiscutível de transplante cardíaco.

Mesmo nos casos de circulação coronária VD-dependente é extremamente rara a documentação de ausência de conexão proximal das artérias coronárias na aorta, o que foi, previamente, documentado, apenas com cateterismo ou necropsia. A documentação adequada de uma situação como esta, mediante ecocardiograma com posterior confirmação angiográfica, é única na literatura nacional e internacional.

\section{Referências}

1. Freedom RM, Harrington DP - Contributions of intramyocardial sinusoids in pulmonary atresia and intact ventricular septum to a right-sided circular shunt. Br Heart J 1974; 36: 1061-5.

2. Calder AL, Co EE, Sage MD - Coronary arterial abnormalities in pulmonary atresia with intact ventricular septum. Am J Cardiol 1987; 59: 436-42.

3. Kasznica J, Ursell PC, Blanc WA, Gersony WM - Abnormalities of the coronary circulation in pulmonary atresia and intact ventricular septum. Am Heart J 1987; 114: $1415-20$

4. Giglia TM, Mandell VS, Connor AR, Mayer Jr JE, Lock JE - Diagnosis and management of right ventricle-dependent coronary circulation in pulmonary atresia with intact ventricular septum. Circulation 1992; 86: 1516-28.

5. Davignon AL, Greenwold WE, Dushane JW, Edwards JE - Congenital pulmo- nary atresia with intact ventricular septum. Clinipathologic correlation of two anatomic types. Am Heart J 1961; 62: 591.

6. Lenox CC, Briner J - Absent proximal coronary arteries associated with pulmonic atresia. Am J Cardiol 1972; 30: 666-9.

7. Blackman MS, Schneider B, SondheimerHM-Absent proximal left main coronary artery in association with pulmonary atresia. Br Heart J 1981; 46: 449-51.

8. Ueda K, Saito A, Nakano H, Hamazaki Y - Absence of proximal coronary arteries associated with pulmonary atresia. Am Heart J 1983; 106: 596-8.

9. Garcia OL, Gelband H, Tamer DF, Fojaco RM - Exclusive origin of both coronary arteries from a hypoplastic right ventricle complicating an extreme tetralogy of Fallot: lethal myocardial infarction following a palliative shunt. Am Heart J 1988; 115: 198-201. 